\title{
Review of: "Ventrolateral prefrontal hemodynamic responses in autism spectrum disorder with and without depression"
}

\author{
Alessia Gallucci ${ }^{1}$ \\ 1 University of Milan - Bicocca
}

Potential competing interests: The author(s) declared that no potential competing interests exist.

In their manuscript entitled "Ventrolateral prefrontal hemodynamic responses in autism spectrum disorder with and without depression", Toshiyuki Ohtani an coauthors present a case-cotrol study exploring differences in brain function between adolescents with autism spectrum disorder and those with comorbid depression. The aim was to point out the existence of a possible biomarker allowing to distinguish comorbid depression that is generally difficult to diagnose in autism.

In my opinion, this is an interesting and timely study. It seems well designed, well conducted and well written in most of its parts. However, I see a series of major issues to be fixed:

1. Authors were not consistent in reporting the clinical assessment they performed. Indeed in the "Participants" section they stated that "Subjects with ASD+D and ASD-D were diagnosed according to the Diagnostic and Statistical Manual of Mental Disorders, 5th Ed. [1], Autism Diagnostic Observation Schedule Second Edition [17], and Autism Diagnostic Interview-Revised [18]. In addition, the Mini International Neuropsychiatric Interview [19] was used to confirm whether there was a current major depressive episode and screen for current and past comorbid psychiatric disorders.". However, these clinical scales are not reported nor described in the "Clinical and neuropsychological assessments" section, in which it is reported that the Wechsler Adult Intelligence Scale 3rd Edition was used to estimate the IQ, the Beck Depression Inventory Scale 2nd edition was used to assess depression, the Autistic-Spectrum Quotient Japanese version for the autistic spectrum traits and the Social Adaptation Self-evaluation Scale to assess the social functioning. I would suggest to solve this issue.

2. Linked to the previous point, beyond the formal inconsistency of information reported, it is not clear how authors performed the diagnosis of autism spectrum disorders and depression. Indeed, using selfreport measures, like the BDI-II and the AQ-J are, could actually lead to misdiagnoses as social desirability mechanisms, among others, could drive participants' responses. Therefore, I suggest Authors to revise this point in the Method part by clarifying whether only self-report tests were used for the diagnoses or whether expert clinicians performed the diagnoses. Moreover, as this issue was highlighted in the limitation section, I suggest Authors to collect another sample of participants screened for autism spectrum and depression symptoms by expert clinicians in order to compare the 
results and verify possible differences based on the diagnosis methodology.

3. Authors considered participants with autism spectrum disorders in general without distinguishing or considering as factors at least the severity and the type of symptoms reported by participants. This could limit the results interpretation. I suggest Authors at least to comment this issue as limitation of their study or, alternatively, to re-rum the analysis by adding the factors I mentioned.

4. Regarding the introduction section, I think that it is not well specified the decision of using the verbal fluency task as cognitive measure. Indeed, it seems that Authors chose this task only because “Many previous fNIRS studies used executive tasks, such as the verbal fluency task (VFT), as a cognitive task to activate regional brain function". In actually, there is a strong literature linking depression with performance on verbal fluency task (see for instance https://scholar.google.com/scholar? as_ylo $=2020 \& q=$ depression+verbal+fluency\&hl=en\&as_sdt=0,5). Therefore, I suggest Authors to revise this part of the introduction section by adding evidence supporting the feasibility of such kind of task to explore makers of depressive symptoms.

5. Authors pointed out in their limitation section that "VFT requires various cognitive functions that may affect the observed results". I think that it is not sufficient to highlight this issue only as limitation. I suggest Authors to collect another sample to compare results of the present study with a control task that not involve executive functions.

6. Regarding the NIRS measurement, Authors considered brain regions that are involved in executive functions and in verbal abilities needed for the verbal fluency task. However, the interpretation of the results could be limited by the absence of a control region. I suggest Authors to collect another another sample to compare results of the present study with a control area. 